\title{
Diagnósticos de Enfermagem do Padrão Mover em idosos de uma comunidade atendida pelo Programa Saúde da Família
}

\author{
NURSING DIAGNOSES OF THE PATTERN OF MOBILITY IN THE ELDERLY \\ ATTENDED BY THE FAMILY HEALTH PROGRAM
}

DIAGNÓSTICOS DE ENFERMERÍA DEL PATRÓN MOVIMIENTO EN ANCIANOS

ASISTIDOS POR EL PROGRAMA SALUD DE LAFAMILIA

\section{Lorena Aparecida de Oliveira Araújo', Maria Márcia Bachion²}

\section{RESUMO}

Com o envelhecimento, eleva-se a ocorrência de incapacidade ou risco para perda da mobilidade nos idosos. Objetivamos estudar a ocorrência de 22 Diagnósticos de Enfermagem do Padrão Moveremidosos, em relação a fre-qüência, sexo e idade. Trata-se de estudo exploratório, que incluiu 75 idosos atendidos por uma equipe do Programa Saúde da Família em Goiânia (GO). Verificamos que $97,3 \%$ da amostra apresentou pelo menos um dos diagnósticos estudados. Os diagnósticos com maiores freqüências foram Mobilidade Física Prejudicada (90,7\%), Padrão do sono perturbado (89,4\%) e Manutenção ineficaz da saúde $(75,9 \%)$. Não encontramos diferença estatisticamente significativa entre a freqüência de diagnósticos entre homens e mulheres. Identificamos diferença estatisticamente significativa entre a freqüência de diagnósticos e a idade. Percebemos que existem características especificas da amostra que sugerem a necessidade de que cada equipe do Programa Saúde da Família avalie sua respectiva clientela a fim de direcionar intervenções precoces e apropriadas.

\section{DESCRITORES}

Enfermagem geriátrica.

Diagnóstico de enfermagem.

Atividade motora.

Programa Saúde da Família.

\begin{abstract}
With aging there is an increase of the occurrence of incapacity and risk of loss of mobility in the elderly. Our objective was to study the occurrence of 22 Nursing Diagnoses of the Moving Pattern in elderly people in relation to frequency, sex and age. It is an exploratory study that involved 75 elders assisted by a team of the Family Health Program in Goiânia, State of Goiás. We found that $97.3 \%$ of the sample presented one or more diagnoses. The most frequent diagnoses were Impaired Physical Mobility (90.7\%), Pattern of the Disturbed Sleep (89.4\%) and Inefficient Health Upkeep $(75.9 \%)$. We did not find statistically significant difference in the frequency of diagnoses among men and women. We identified statistically significant difference between the frequency of diagnoses and age. We noticed that there are specific characteristics of the sample that suggest the need for each team of Health Family Program to evaluate its clientele in order to direct early appropriate interventions.
\end{abstract}

\section{KEY WORDS}

Nursing diagnosis.

Geriatric nursing.

Motor activity.

Family Health Program.

\section{RESUMEN}

Con el envejecimiento, aumenta la ocurrencia de incapacidad o el riesgo para la pérdida de la movilidad en los ancianos. Nuestro objetivo fue estudiar la ocurrencia de 22 Diagnósticos deEnfermería del patrón movimiento en los ancianos, en relación a la frecuencia, sexo y edad. Se trata de uno estudio exploratorio que incluyó a 75 ancianos, asistidos por una equipo del Programa Salud de la Familia en Goiania (Brasil). Verificamos que el 97,3\% de la muestra presentó porlomenos un diagnóstico. Los diagnósticos con mayores frecuencias fueron: movilidad física perjudicada $(90,7 \%)$, modelodel sueño alterado $(89,4 \%)$ y manutención ineficaz de la salud $(75,9 \%)$. No encontramos diferencia estadísticamente significativa entre la frecuencia de los diagnósticos entre hombres y mujeres. Identificamos diferencia estadísticamente significativa entre la frecuencia de los diagnósticosy la edad. Percibimos que existen características específicas de la muestra que sugieren la necesidad dequecadaequipodelProgramaSalud de la Familia evalúe su respectiva clientelaa fin de orientarintervenciones precoces y apropiadas.

\section{DESCRIPTORES}

Diagnóstico de enfermería.

Actividad motora.

Enfermería geriátrica.

Programa Salud de la Familia.
1 Enfermeira do Programa Saúde da Família. Secretaria Municipal de Saúde. Aluna do Programa de Pós-Graduação em Ciências da Saúde, Convênio UNB/UFG/UFMS

2 Enfermeira, Doutora em Enfermagem. Professor Titular da Faculdade de Enfermagem da Universidade Federal de Goiás.amm03@popmail.com 
Lorena A. de O. Araújo

Maria Márcia Bachion

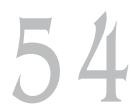

Rev Esc Enferm USP 2005; 39(1):53-61.

\section{INTRODUÇÃO}

No Brasil, a expectativa de vida no último século aumentou de 34 para 66 anos, podendo chegar a 72 anos em 2020. A população idosa em vinte anos $(1980-2000)$ cresceu $86,7 \% \%^{(1)}$ e atualmente equivalem a $7,8 \%$ da população total, o que representa aproximadamente 14 milhões de pessoas com 60 anos ou mais. Em Goiás esta taxa é de $6,25 \%$, ou seja, aproximadamente 338.000 pessoas. Goiânia possui cerca de um milhão de habitantes. Nesse contingente, o grupo de idosos representa $6,16 \%$ da população predominando aqueles com 60 a 69 anos (3,7\%), seguindo-se por idosos com idade entre 70 e 79 anos $(1,73 \%)$ e, por fim, maiores de 80 anos que equivalem a $0,65 \%$ da população total da cidade ${ }^{(2)}$.

É notório que a medida em que se envelhece aumenta a prevalência de doenças crônicas, que levam à maior parte da ocorrência de incapacidade nos idosos. É comum que o ser humano tenha pelo menos uma patologia crônico-degenerativa após os 60 anos de idade, sendo que pelo menos $15 \%$ destas pessoas apresentem duas ou mais doenças ${ }^{(1)}$, que por sua vez, podem provocar algum tipo de incapacidade e/ou dependência ${ }^{(3)}$.

Nesse sentido os profissionais de saúde são desafiados a lidar com uma prevalência mais alta de doenças que ocorrem nesta população, onde muitos distúrbios crônicos comuns podem ser controlados, limitados e até mesmo prevenidos, assim as pessoas idosas podem ter maior probabilidade de manter uma boa saúde e independência funcional ${ }^{(4)}$. A assistência ao idoso deve prezar pela manutenção da qualidade de vida, considerando os processos de perdas próprias do envelhecimento e as possibilidades de prevenção, manutenção e reabilitação de seu estado de saúde ${ }^{(5)}$.

Problemas relacionados à área motora em idosos são do tipo potencial, ou seja, a população idosa tem risco para perda da mobilidade ${ }^{(6)}$. Durante o processo de envelhecimento fisiológico dentre as inúmeras modificações que ocorrem, assinalamos aquelas relativas à atividade motora, como o enfraquecimento muscular e músculoesquelético, diminuição da coordenação motora e equilíbrio, perda de massa muscular, tônus muscular diminuído entre outras alterações ${ }^{(4,6)}$.

Outro aspecto importante a ser ressaltado é a possível perda da autonomia a que o idoso está sujeito, visto que qualquer "déficit" na área motora, pode afetar suas escolhas na tomada de decisões referentes a suas atividades da vida diária e instrumentais.
Todavia é importante evidenciar que o declínio das funções orgânicas pode variar consideravelmente entre os idosos, podendo ser muito acentuado para uns e insignificante para outros, mesmo na oitava década da vida ${ }^{(1)}$.

A Enfermagem ainda é deficitária para oferecer assistência especializada aos idosos, sendo necessário qualificar os profissionais, criar novos espaços de atendimento para esta clientela e novas abordagens teóricas. O Processo de Enfermagem tem representado o principal instrumento metodológico para o desempenho sistemático da prática profissional. Consiste de fases interdependentes e inter-relacionadas, nas quais ocorre a coleta e análise dos dados e identificação dos possíveis diagnósticos de enfermagem, planejamento da assistência a ser prestada, implementação de ações e/ ou intervenções e avaliação dos resultados alcançados.

Um dos sistemas de classificação de diagnósticos, a taxonomia da North American Nursing Diagnoses Association (NANDA), define Diagnóstico de Enfermagem (DE) como sendo

um julgamento clínico das respostas do indivíduo, da família ou da comunidade a problemas de saúde/processos vitais reais ou potenciais, proporcionando base para a seleção de intervenções de enfermagem para atingir resultados pelos quais a enfermeira é responsável(7).

Nesta taxonomia são propostos nove padrões que manifestam a saúde do cliente se estiverem em total equilíbrio: trocar, comunicar, valorizar, escolher, relacionar, mover, perceber, conhecer, e sentir. Enfermeiros de todo o mundo têm utilizado essa classificação no cuidado, no ensino e na pesquisa. Este sistema de classificação inclui título dos diagnósticos, definição, fatores relacionados e características definidoras.

O fator relacionado é conceituado como o conjunto de fatores, fisiológicos, psicológicos, socioculturais, ambientais ou espirituais que possam estar ocasionando ou contribuindo para uma reação no cliente ${ }^{(8)}$. As características definidoras por sua vez são dados objetivos e subjetivos, sinais e sintomas que indicam a presença de um DE. ${ }^{(7-8)}$.

Em estudo realizado anteriormente, abrangendo idosos institucionalizados verificamos que a totalidade dos sujeitos apresentava o diagnóstico de enfermagem mobilidade física prejudica$\mathrm{da}^{(9-10)}$. Entre os fatores relacionados (causas ou 
fatores coadjuvantes) identificados como os mais freqüentes estavam enfraquecimento músculoesquelético, força e resistência diminuídas, enfraquecimento neuromuscular e dano perceptual ou cognitivo. Em outra pesquisa, considerando idosos de um condomínio residencial filantrópico, identificamos 96\% dos moradores com Mobilidade física prejudicada, sendo que os fatores relacionados apresentados com maior incidência neste grupo foram o enfraquecimento músculoesquelético, enfraquecimento neuromuscular, dano perceptual ou cognitivo e depressão ou ansiedade severa $^{(11)}$.

Acreditando que problemas relacionados à mobilidade física são de grande influência no estado de saúde dos idosos, já que no decorrer do processo de envelhecimento físiológico esta população sofre modificações significativamente importantes na área, optamos por seu estudo, em idosos que vivem em uma comunidade atendida por uma equipe do Programa Saúde da Família em Goiânia(GO).

O Programa Saúde da Família (PSF) é uma estratégia do Ministério da Saúde com o objetivo de reorganizar a prática assistencial em novas bases e critérios, priorizando as ações de prevenção de doenças e promoção a saúde junto à comunidade, estabelecendo uma relação permanente entre os profissionais de saúde e a população assistida, com vistas a um atendimento humanizado e resolutivo dos problemas de saúde mais freqüentes ${ }^{(12)}$.

\section{OBJETIVOS}

O objetivo geral deste estudo é verificar a ocorrência de 22 Diagnósticos de Enfermagem do Padrão Mover em idosos cadastrados por uma equipe do Programa Saúde da Família de Goiânia (GO). Neste sentido, estabelecemos como objetivos específicos:

- Identificar a freqüência de 22 Diagnósticos de Enfermagem do Padrão Mover em idosos.

- Comparar a distribuição da freqüência dos Diagnósticos de Enfermagem estudados entre os gêneros masculino e feminino;

- Comparar a distribuição da freqüência dos Diagnósticos de Enfermagem do Padrão Mover entre diferentes faixas etárias.

\section{CASUÍSTICA E MÉTODO}

Trata-se de um estudo exploratório, transversal, cuja população foram pessoas com idade mai- or ou igual à 60 anos, moradores do Jardim Mariliza em Goiânia (Região/Distrito Sudeste), atendidos pela equipe 02 do Programa Saúde da Família e que se dispuseram a participar. Utilizamos, para a análise de dados, procedimentos de estatística descritiva, tais como a distribuição por freqüência simples de ocorrência de patologias. Utilizamos ainda o programa estatístico Statistical Program of Social Sciences (SPSS) para análise de dados referente aos Diagnósticos de Enfermagem (Padrão Mover em idosos), comparando sua ocorrência entre os gênero e as faixas etárias.

$\mathrm{O}$ projeto de pesquisa foi encaminhado à $\mathrm{Fa}-$ culdade de Enfermagem da Universidade Federal de Goiás, Coordenação Colegiada do Programa Saúde da Família da Secretaria de Saúde de Goiânia, para apreciação, bem como ao Comitê de Ética em Pesquisa da Universidade Federal de Goiás. Após a aprovação do projeto de pesquisa em todas as instâncias foi iniciada a abordagem dos idosos. Ao aceitar participar do estudo, os sujeitos assinaram termo de consentimento livre e esclarecido, sendo garantido sigilo e anonimato aos participantes, atendendo a Resolução 196/96 do Conselho Nacional de Saúde ${ }^{(13)}$.

Foram utilizadas na pesquisa as duas primeiras fases do Processo de Enfermagem, ou seja, a Coleta de Dados e o Diagnóstico de Enfermagem. Utilizamos como instrumento de coleta de dados uma adaptação do protocolo de avaliação clínica de Mobilidade Física Prejudica adotada em estudos anteriores $^{(9-11)}$. Este roteiro prevê procedimentos de entrevista e exame físico. A coleta de dados ocorreu em ambiente privativo, no domicílio do idoso ou no consultório de enfermagem do PSF do setor.

\section{RESULTADOS E DISCUSSÃO}

Entre os 103 idosos cadastrados pela equipe 02 do Programa Saúde da Família do Jardim Mariliza, 75 aceitaram participar deste estudo, sendo que destes, $32 \%$ têm idade entre 60 e 64 anos, $12 \%$ têm entre 65 e 70 anos, 13,3\% indivíduos estão na faixa etária de 71 a 75 anos, $14,6 \%$ possuem entre 76 e 79 anos, $13,3 \%$ idosos apresentam entre 80 e 84 anos e 5,3\% sujeitos são maiores de 85 anos. A média de idade foi de 71,05 anos, sendo que para os homens encontramos 71,67 e para as mulheres 70,76 anos.

Quanto às patologias relatadas (doenças previamente identificadas por profissionais médicos), os idosos referiram hipertensão arterial $(57,3 \%)$, reumatismo $(25,3 \%)$, osteoporose $(24 \%)$, labirintite
Diagnósticos de Enfermagem do Padrão Mover em idosos de uma comunidade atendida pelo Programa Saúde da Família 
Lorena A. de O. Araújo Maria Márcia Bachion
$(21,3 \%)$, diabetes $(20 \%)$, depressão (12\%) e outros, com ocorrência menor, como seqüela de acidente vascular cerebral, bursite, doença de Parkinson, doença de Chagas. Em média os idosos relataram entre 2 e 3 patologias $(2,61)$.

Em São Paulo apenas 14\% dos idosos referiram estar livres de doenças crônicas ${ }^{(1)}$, enquanto em nossa amostra encontramos este relato em $4 \%$ dos idosos. Por outro lado nessa pesquisa realizada em São Paulo, 15 \% mencionam cinco ou mais patologias de natureza crônica degenerativa ${ }^{(1)}$. Em nosso estudo tal inferência foi relatada por $10,6 \%$ dos idosos.

A HAS foi referida por 43 (57,3\%) idosos. Este achado assume grande relevância na perspectiva de que a hipertensão é um fator de risco importante para as doenças cardiovasculares e corrobora para a afirmação de que, embora constitua um problema característico da população ido$\mathrm{sa}^{(4)}$, a literatura aponta que aproximadamente $40 \%$ dos indivíduos maiores de 65 anos são hipertensos ${ }^{(14)}$.

Os problemas relacionados aos diversos tipos de reumatismos (artrite, artrose, poliartrite reumática) afetam cerca de $50 \%$ da população idosa, sendo uma importante causa de sofrimento, restrição de atividades e utilização dos serviços de saúde ${ }^{(6)}$. Nesta comunidade tal característica foi encontrada em 19 (25,3\%) indivíduos.

A osteoporose foi encontrada em 18 (24\%) idosos. Os ossos tornam-se progressivamente mais porosos e frágeis, percebidos principalmente em mulheres menopausadas ${ }^{(6)}$.

A obesidade foi identificada em 22,6\% dos idosos da amostra o que representa um problema importante a medida que esta condição é fator de risco para outras doenças e a maior parte dos idosos não modificam os seus hábitos alimentares mesmo quando aumentam o peso ${ }^{(6)}$.

Para verificarmos a existência de depressão nestes idosos utilizamos a Escala Geriátrica de Yesavage $^{(14)}$. Detectamos que 70,7\% não apresentavam tal ocorrência, 24\% idosos apresentam depressão moderada, segundo a escala e 5,3\% clientes apresentam depressão grave. Contudo, apenas 09 idosos haviam-se referido à depressão como diagnóstico médico.

Alguns trabalhos relatam que a depressão nos idosos integrados a comunidade tem uma incidência (números de casos novos ao ano) aproximada de $2 \%$ e de prevalência (número de casos novos no ano mais os já existentes) de $10 \%$ e dessa porcentagem cerca de $2 \%$ são depressões $\operatorname{graves}^{(6,14)}$.

O diabetes mellitus foi relatado por $15(20 \%)$ indivíduos da amostra. Entre as pessoas com mais de 65 anos de idade, 8,6\% tem diabetes tipo II ${ }^{(1)}$.

Entre os idosos que apresentaram diagnósticos de enfermagem no padrão estudado, foram identificadas de zero a 18 ocorrências, sendo que esta última quantidade foi encontrada em um idoso em processo demencial com HAS e Diabetes.

A média de diagnósticos por idosos foi igual a 7,43 (6,42 para os homens e 7,9 para as mulheres). Considerando os participantes no geral, a moda de diagnósticos encontrada foi igual a 3 .

O diagnóstico de enfermagem "mobilidade física prejudicada" definido como "limitação do movimento físico independente e voluntário do corpo ou de uma ou mais extremidades"(8), foi observado em $92 \%$ dos idosos, conforme mostra a tabela 1. Esse diagnóstico não necessariamente está relacionado à incapacidade, mais sobretudo a fisiologia do envelhecimento, a falta de atividade física regular, a sensação dolorosa e a hábitos de vida.

Prosseguindo, discutiremos os demais diagnósticos de acordo com a ordem por afinidade da natureza da resposta humana.

Capacidade diminuída para andar, definida como "limitação do movimento independente, a pé, pelo ambiente" ${ }^{(8)}$, foi encontrada em $44 \%$ da amostra. Os elementos que compõem a caminhada são amplitude de movimento, equilíbrio, força muscular, coordenação motora e todos são afetados pelo processo de envelhecimento ${ }^{(6)}$.

Assim, são necessárias ações dos profissionais de saúde para estimular o desenvolvimento de atividades como caminhadas, jogos, hidroginástica entre outros, além de medidas de adequação do urbanismo para facilitar a performance dessa população, paciência dos mais jovens, incluindo os profissionais de saúde, para respeitar a velocidade de deslocamento dessas pessoas.

O uso de cadeira de rodas foi detectado em apenas um (1,3\%) dos participantes deste estudo, decorrente de hemiparesia direita causada por acidente vascular cerebral. Apesar do acompanhamento fisioterápico e da prática de hidroginástica, não houve remissão do quadro. O mesmo apresentou "mobilidade de cadeiras de 
rodas prejudicada", definida como "limitação da operação com cadeira de rodas pelo ambiente"(8). Para operar com eficiência uma cadeira de rodas o indivíduo deve ter destreza, força, coordenação motora, equilíbrio de tronco, flexibilidade e amplitude de movimentos eficazes em membros superi- ores. Uma vez que estas habilidades podem estar diminuídas nos idosos, para aqueles que são usuários de cadeira de rodas (cadeirantes) é necessário que a cadeira seja da melhor qualidade possível para favorecer o deslocamento.

Tabela 1- Diagnósticos de Enfermagem do Padrão Mover em idosos, de acordo com o sexo. Equipe 2, PSF/Jardim Mariliza. (Goiânia (GO), 2003)

\begin{tabular}{|c|c|c|c|c|c|c|}
\hline \multirow[t]{2}{*}{ Diagnóstico de Enfermagem } & \multicolumn{2}{|c|}{$\begin{array}{c}\text { Feminino } \\
(f=51)\end{array}$} & \multicolumn{2}{|c|}{$\begin{array}{l}\text { Masculino } \\
\quad(f=24)\end{array}$} & \multicolumn{2}{|c|}{$\begin{array}{c}\text { Total } \\
(f=75)\end{array}$} \\
\hline & $\%$ & $(f)$ & $\%$ & $(f)$ & $\%$ & $(f)$ \\
\hline Mobilidade física prejudicada & 92,2 & $(47)$ & 91,7 & $(22)$ & 92 & (69) \\
\hline Padrão do sono perturbado & 88,2 & (45) & 87,5 & (21) & 88 & (66) \\
\hline Manutenção ineficaz da saúde & 80,4 & $(41)$ & 66,7 & (16) & 75,9 & (57) \\
\hline Fadiga & 54,9 & $(28)$ & 33,3 & $(8)$ & 48 & (36) \\
\hline Intolerância a atividades físicas & 52,9 & $(27)$ & 29,2 & (7) & 45,3 & $(34)$ \\
\hline Capacidade diminuída para andar & 51 & (26) & 29,2 & (7) & 44 & (33) \\
\hline Manutenção do lar prejudicada & 47,1 & $(24)$ & 37,5 & (9) & 44 & (33) \\
\hline Falha do adulto em melhorar & 33,3 & (17) & 33,3 & (8) & 33,3 & (25) \\
\hline Deglutição prejudicada & 39,2 & $(20)$ & 20,8 & (5) & 33,3 & (25) \\
\hline Atividades de recreação deficientes & 35,3 & (18) & 29,2 & (7) & 33,3 & (25) \\
\hline Privação do sono & 31,4 & (16) & 29,2 & (7) & 30,6 & (23) \\
\hline Déficit no autocuidado para alimentação & 25,5 & (13) & 33,3 & (8) & 28 & (21) \\
\hline Risco para intolerância a atividades físicas & 29,4 & (15) & 16,7 & (4) & 25,3 & (19) \\
\hline Déficit autocuidado para banho/ higiene & 25,5 & (13) & 25 & (6) & 25,3 & (19) \\
\hline Capacidade de transferência prejudicada & 23,5 & $(12)$ & 12,5 & (3) & 20 & (15) \\
\hline Déficit autocuidado vestir-se/ arrumar-se & 21,6 & (11) & 12,5 & (3) & 18,6 & (14) \\
\hline Síndrome de estresse por mudança & 17,6 & (9) & 16,7 & (4) & 17,3 & (13) \\
\hline Risco disfunção neurovascular periférica & 11,8 & (6) & 12,5 & (3) & 12 & (9) \\
\hline Mobilidade no leito prejudicada & 15,7 & (8) & 12,5 & (3) & 10,7 & (11) \\
\hline Déficit autocuidado para higiene íntima & 5,4 & (3) & 12,5 & (3) & 8 & (6) \\
\hline Recuperação cirúrgica retardada & 1,9 & (1) & 8,3 & (2) & 4 & (3) \\
\hline Nenhum diagnóstico encontrado & 1,9 & (1) & 4 & (1) & 2,7 & (2) \\
\hline Mobilidade em cadeiras de rodas prejudicada & 1,9 & $(1)$ & 0 & $(0)$ & 1,3 & (1) \\
\hline
\end{tabular}

Capacidade de transferência prejudicada, definida como"limitação ao movimento entre duas superfícies próximas"(7), foi observada em $20 \%$ dos idosos desta comunidade. Este diagnóstico foi avaliado em todos os idosos não somente aqueles que faziam uso de cadeira de rodas visto que mesmo os não cadeirantes podem-se transferir de posições, por exemplo, da posição de pé para a sentada ou de pé para o chão etc.

Mobilidade no leito prejudicada, que se define pela "limitação ao movimento independente de uma posição para outra no leito"(7) foi observada em $10,7 \%$ dos idosos. Tal diagnóstico pode ser observado quando o indivíduo apresenta dificuldades em levantar-se da cama, sentar-se sem apoio, realizar independentemente mudança de decúbito.

Intolerância a atividade física, que é a "insuficiência de energia fisiológica ou psicológica para suportar ou completar as atividades diárias requeridas ou desejadas"(7) foi observada em $45,3 \%$ da amostra. Risco para intolerância a atividade física, quer dizer "estar em risco de experimentar a insuficiência de energia físiológica ou psicológica para suportar ou completar as atividades diárias requeridas ou desejadas"(7) foi observado em $25,3 \%$ dos idosos.

A intolerância a atividade física, provém muitas vezes de uma falta de motivação na seqüência de uma imobilização prolongada ou de uma patologia cardiorrespiratória ${ }^{(6)}$. Certas patologias, incluindo as das artérias coronárias, vasculares periféricas, arritmias cardíacas, insuficiência cardíaca congestiva, doenças pulmonares crônicas e pneumonias reduzem a oxigenação dos tecidos e, por conseguinte, a tolerância a atividade, sendo legítimo dizer que uma pessoa com intolerância a atividade tenha ou poderá vir a ter alterações na mobilidade e que uma pessoa como problema de mobilidade poderá vir a ter intolerância.

Déficit no autocuidado para banho/ higiene, ou seja, "capacidade prejudicada de realizar completar as atividades de banho/higiene por si mesmo"(7) foi encontrada em $25,3 \%$ da comunidade estudada. Déficit no autocuidado para higiene íntima, definida como a "capacidade prejudicada de realizar suas próprias atividades de higiene ínti-
Diagnósticos de Enfermagem do Padrão Mover em idosos de uma comunidade atendida pelo Programa Saúde da Família 
Lorena A. de O. Araújo Maria Márcia Bachion ma"(8) foi encontrada em $8 \%$ da amostra. A independência na satisfação da necessidade de estar limpo, cuidado e proteger os tegumentos permite ao ser humano manter a saúde física e emocional. O significado da necessidade de limpeza e os meios utilizados para a satisfação dessa necessidade variam em função dos indivíduos ${ }^{(6)}$.

Devemos estar atentos em relação a alguns aspectos da avaliação do idoso, especialmente quando se trata de condições de desempenho ou execução das atividades de vida diária (AVD) e instrumentais de vida diária (AIVD), sendo indispensável à determinação de seu grau de autonomia e de independência levando sempre em consideração o seu conforto, segurança e bemestar, até que as AVD e AIVD possam ser restauradas quando afetadas ${ }^{(15)}$.

Déficit no autocuidado para vestir-se/ arrumar-se, que é a "capacidade prejudicada de reali$\mathrm{zar} /$ completar atividades de vestir-se e arrumarse por si mesmo" ${ }^{(7)}$ foi encontrado em 18,6\% dos entrevistados.

O vestuário desempenha um papel primordial no bem-estar psicológico do indivíduo, sendo várias vezes utilizado como meio adaptativo a necessidades ambientais e sociais, constituindo um valor importante para os idosos, devido à perda dos suportes habituais de ego (vigor físico e status social). Vestir-se e despir-se exigem muita coordenação, destreza, equilíbrio, amplitude de movimento e força muscular e como estas funções estão afetadas pelo envelhecimento do sistema músculo-esquelético, os idosos por vezes apresentam dificuldade em realizar tal ação( ${ }^{(6)}$.

Manutenção do lar prejudicada, compreendida como a "capacidade de manter de forma independente um ambiente imediato, seguro e pré promova o crescimento" ${ }^{(7)}$ tendo sido referido em $44 \%$ dos clientes. Em geral os idosos destacaram dificuldades em varrer e passar pano no chão da casa atividades que exigem destreza, força muscular, equilíbrio e amplitude de movimento.

Nesta amostra, 33,3\% apresentou Atividades de recreação deficientes, definida como "estimulação, interesse ou engajamento diminuídos em atividades recreativas ou lazer"'(7). Distrair-se é uma necessidade do todo o ser humano, e aquele que se diverte com uma ocupação agradável com fim de se descontrair física e psicologicamente satisfaz esta necessidade fundamental, sobretudo as pessoas idosas devem ter esta necessidade de realizar as atividades recreativas suprida à medida em que suas capacidades lhe permitirem envelhecimento ${ }^{(6)}$. Muitas atividades recreativas exigem coordenação, destreza e força muscular, e como estas funções são afetadas pelo processo de envelhecimento, os idosos têm dificuldade em praticar suas atividades habituais deslocando geralmente o foco físico (esportes, dança) para o foco intelectual (leitura, palavra-cruzada, jogos) envelhecimento ${ }^{(6)}$.

Déficit no autocuidado para alimentação ("capacidade de desempenhar ou completar atividades de alimentação") ${ }^{(7)}$ foi encontrado em $28 \%$ da amostra. A alimentação é um fator primordial para a promoção, manutenção e/ ou recuperação da saúde em todas as fases da vida e que com o processo de envelhecimento, as pessoas podem perder o interesse em preparar e ingerir as refeições ${ }^{(16)}$.

Outras alterações relacionadas ao envelhecimento como mudanças fisiológicas no trato gastrintestinal, fatores sociais e econômicos, interações de drogas e doenças, uso excessivo de bebidas alcoólicas, dentição em mau estado ou ausência ${ }^{(4)}$.

Deglutição prejudicada, ou seja, o "funcionamento anormal do mecanismo de deglutição associado com déficit na estrutura ou na função oral, faríngea ou esofágica"(7) foi encontrada em $33,3 \%$ dos idosos. Dificuldade de deglutição é freqüente nos idosos que engasgam com facilidade com os alimentos, devido à diminuição da motilidade digestiva por perda de acuidade neuromuscular. Muitas vezes, a porção inferior do esôfago apresenta-se dilatada, o que desencadeia espasmos, refluxo digestivo e dor ${ }^{(6)}$.

Manutenção ineficaz da saúde, quer dizer, "capacidade de identificar, controlar e/ ou buscar ajuda para manter a saúde"(7) foi encontrada em $75,9 \%$ dos idosos desta comunidade. No que diz respeito à identificação deste diagnóstico a maioria dos indivíduos da amostra destaca a falta demonstrada de conhecimento com relação a práticas básicas de saúde, em especial na ida ao dentista. Muitos dos clientes utilizam próteses dentárias acreditando que "como os dentes não são mais os meus, não há necessidade de irem ao especialista".

Falha do adulto em melhorar compreende "deterioração funcional progressiva de natureza física e cognitiva", assim a "capacidade de viver com doenças multissistêmicas, enfrentar os problemas decorrentes e controlar o seu cuidado está notavelmente diminuída" ${ }^{(7)}$. Este fenômeno foi detectado em $33,3 \%$ dos entrevistados, sendo 
caracterizado pela exacerbação freqüente de problemas de saúde (como pneumonia e infecção do trato urinário), ingesta nutricional inadequada comer menos dos que as necessidades corporais, declínio cognitivo nos últimos seis meses.

Recuperação cirúrgica retardada, definida como a "extensão do número de dias de pós-operatório necessários para iniciar e desempenhar atividades que mantêm a vida, a saúde e o bem estar"(7) foi relatada por $4 \%$ dos clientes, contudo é importante destacar que outros clientes inferiram ter sofrido processo cirúrgico, contudo somente 2 indivíduos afirmam não estarem recuperados em sua totalidade, tendo como queixa principal dor ou desconforto no local da cirurgia.

Risco para disfunção neurovascular periférica, compreendido como "estar em risco de distúrbio na circulação, na sensibilidade ao movimento de uma extremidade" ${ }^{\text {(8) }}$, foi encontrado em $12 \%$ da amostra. Uma quantidade menor de suprimento sangüíneo decorrente de um distúrbio circulatório coloca a pessoa idosa em grande risco de ser acometido por infecções em regiões periféricas e outros problemas subseqüentes ${ }^{(4)}$.

Síndrome de estresse por mudança, que é o "distúrbio fisiológico e/ ou psicossociais decorrentes de mudança de um ambiente para outro"(7), foi relatada por $17,3 \%$ dos idosos desta comunidade que afirmam dificuldades quanto à adaptação a mudança de endereço, seja por afastamento de pessoas queridas, por localização ou por acesso a recursos da comunidade como farmácias e supermercados.

Fadiga, isto é, a "sensação opressiva e sustentada de exaustão e de capacidade diminuída para realizar trabalho físico e mental no nível habitual"(7), foi observada em $48 \%$. A fadiga pode ser um indicador de depressão ou um sintoma de doença física como anemia ou cardiopatia, sendo várias vezes caracterizada por aumento das queixas físicas, falta de energia ou incapacidade de manter o nível habitual de atividade física ${ }^{(4)}$.

Privação do sono, quer dizer, "períodos prolongados de tempo sem sono, suspensão sustenta natural e periódica do estado de consciência relativa"(7), foi observado em $30,6 \%$ dos sujeitos.

O sono é um estado de consciência alterado ou um estado de consciência parcial do qual uma pessoa pode sair mediante estímulo adequado ${ }^{(6)}$. O envelhecimento afeta a qualidade de sono ou o nível de repartição dos vários estágios do sono. Problemas como inatividade, doenças, medicamentos, depressão e hábitos pessoais podem agravar os problemas de sono em pessoas idosas ${ }^{(17)}$.
Padrão de sono perturbado, definido como "distúrbio decorrente do tempo limitado na qualidade de sono - suspensão natural, periódica da consciência"(7) foi encontrado em $88 \%$ da amostra.

Tradicionalmente para enfermagem, o sono é considerado uma necessidade humana básica, sendo um dos fatores que influenciam na qualidade de vida do ser humano, contudo nos idosos algumas alterações fisiológicas alteram ou interferem nessa necessidade básica como, por exemplo, diminuição da duração relativa e absoluta do sono profundo e conseqüente aumento do sono superficial, diminuição da duração do sono e sua interrupção várias vezes durante a noite etc ${ }^{(17)}$.

Em nossa amostra apenas dois clientes não apresentaram nenhum dos diagnósticos de enfermagem no Padrão Mover. Trata-se de um homem de 65 anos, aposentado que realiza as tarefas domésticas, é casado e a esposa é trabalhadora ativa. Este idoso tem vida social intensa, pratica atividades físicas regulares em academia de ginástica há três anos. A outra participante é dona de casa ativa, casada e faz todas as atividades do lar independentemente, mesmo apresentado dores leves, ocasionais pelo corpo devido a osteoporose diagnosticada recentemente.

Comparando a prevalência dos diagnósticos estudados entre os gêneros, podemos perceber que as mulheres são mais acometidas por danos na mobilidade, tratando-se em sua maior parte de donas de casa, enquanto que os homens exerciam atividade que exigiam maior esforço físico.

Observando a distribuição da prevalência dos 22 diagnósticos entre homens e mulheres, identificamos apenas 04 com ocorrência maior em homens que em mulheres (Déficit no alto cuidado para alimentação, Síndrome de estresse por mudanças, Recuperação cirúrgica retardada e Déficit no alto cuidado para higiene íntima), contudo não existe diferença estatística significativa entre a média de diagnósticos de enfermagem e o sexo, segundo teste exato de Fischer, sendo $\mathrm{p}=0,05$.

No que se refere à relação entre o total de diagnósticos encontrados e a idade, após realizar o teste Kolmogorov-Smirnov e constatar que esta relação obedece a curva normal, adotamos o Teste $\mathrm{T}$ de Student para análise e $\mathrm{p}=0,05$, identificando que a quantidade total de diagnósticos de enfermagem (TOTAL_DE) aumenta em relação a idade, como podemos observar na figura 1 .

Como todas as necessidades fundamentais, a de mover-se compreende quatro dimensões específicas: a biofísiológica (os subsistemas cardiovas-
Diagnósticos de Enfermagem do Padrão Mover em idosos de uma comunidade atendida pelo Programa Saúde da Família 
Lorena A. de O. Araújo Maria Márcia Bachion cular, músculo e esquelético, nervoso, etc. influenciam a liberdade de se movimentar e adaptarse), a psicológica (estado psicológico como influência no mover-se através da ansiedade, da depressão, da rigidez de pensamento etc.), a sociológica (disponibilidade de rede social de suporte, fatores ambientais etc.), cultural/espiritual (a cultura e a religiosidade influenciam na vida cotidiana do indivíduo, sofrendo interferência dos costumes e tradições $)^{(6)}$.

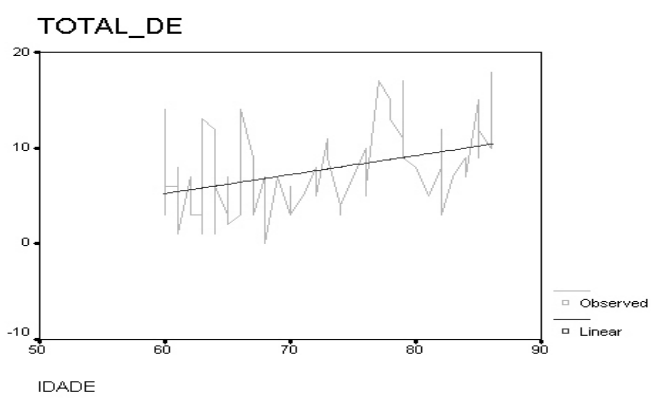

Figura 1- Relação entre o Número de Diagnósticos de Enfermagem (DE) e idade dos idosos. Goiânia (GO), 2003

Dada a magnitude da apresentação de problemas relacionados à mobilidade nesta comunidade, podemos afirmar tratar-se de uma alteração que pode ser considerada questão de saúde coletiva, fazendo-se necessária a elaboração de ações de enfermagem no contexto da atuação de equipe multiprofissional, que possam minimizar, tratar ou sanar o problema. Acreditamos que sejam igualmente importantes ações simultâneas de outros profissionais da área da saúde como psicólogos, fonoaudiólogos, fisioterapeutas, nutricionistas, assistentes sociais, médicos de várias especialidades.

Em estudos realizados com idosos que vivem em instituição $\operatorname{asilar}^{(10)}$ e idosos que vivem em um condomínio residencial ${ }^{(11)}$ foram realizadas intervenções referentes à Mobilidade física prejudicada, obtendo resultados positivos em relação à melhora da capacidade motora dos participan-

\section{REFERÊNCIAS}

(1) Lessa I. O adulto brasileiro e as doenças da modernidade: epidemiologia das doenças crônicas transmissíveis. Rio de Janeiro: Hucitec; 1998.

(2) Ministério da Saúde - Sistema Único de Saúde. Dados de saúde 2000. [online] Brasília; 2004. Disponível em: $<$ http:/ www.datasus.gov.br/> (20 out. 2003). tes, mediante atuação sistemática durante aproximadamente catorze semanas.

Acreditamos que pesquisas como estas devam ser replicadas com a finalidade de desenvolver conhecimentos e habilidades visando à ampliação de um conjunto de intervenções de enfermagem baseado em evidencias seguras para sua aplicação.

\section{CONCLUSÕES}

Dos 75 idosos participantes, 73 (97,3\%) apresentaram pelo menos um dos 22 diagnósticos de enfermagem do Padrão Mover. Os diagnósticos de maior ocorrência foram Mobilidade Física Prejudica $(90,7 \%)$, Padrão do sono perturbado $(89,4 \%)$ e manutenção ineficaz da saúde $(74,7 \%)$.

A Manutenção do lar prejudicada (44\%), Déficit no autocuidado para alimentação (29,3\%), Déficit no autocuidado para banho/ higiene $(25,3 \%)$, Déficit no autocuidado para vestir-se arrumar-se (18,6\%), Déficit no autocuidado para higiene íntima $(5,3 \%)$ remetem ao comprometimento das atividades de vida diária e atividades instrumentais de vida diária.

As mulheres apresentaram em média 7,9 diagnósticos e os homens 6,42. Contudo, de acordo com o teste exato de Fisher $(p=0,05)$ esta diferença não é estatisticamente significativa.

Encontramos uma diferença estatisticamente significativa entre o número de diagnósticos de enfermagem e a idade, sendo que a medida em que a idade aumenta, aumenta também o número de diagnósticos.

Estes resultados remetem à necessidade de intervenções precoces, de natureza interdisciplinar, tanto de reabilitação quanto de prevenção, no âmbito do Programa Saúde da Família junto a esta comunidade, a fim de desenvolver/ melhorar a força muscular, flexibilidade, coordenação, destreza e equilíbrio destes idosos, além da manutenção eficaz da saúde especialmente com relação à saúde bucal. 
(4) Smeltezer SC, Bare BG, editores. Tratado de enfermagem médico-cirúrgica. Trad. de Catarina Salvador

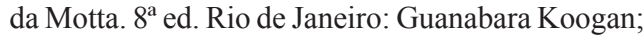
1998. 2v.

(5) Marin MJS, Barbosa PMK, Takitane MT. Diagnósticos de enfermagem mais freqüentes entre idosas hospitalizadas em unidade de clínica médica e cirúrgica. Rev Bras Enferm 2000; 53(4):513-23.

(6) Berger LM, Mailloux-Poirier M. Pessoas idosas: uma abordagem global. Lisboa: Lusodidacta; 1995.

(7) North Americam Nursing Diagnosis Association (NANDA). Diagnósticos de enfermagem da NANDA: definições e classificação 2001-2002. Tradução Janne Liliane Marlena Michael Porto Alegre: Artmed; 2002.

(8) Carpenito LJ. Diagnóstico de enfermagem: aplicação à prática clínica. $6^{\circ}$ ed. Porto Alegre: Artes Médicas; 1997.

(9) Bachion MM, Araújo LAO, Santana RF. Validação de conteúdo "Mobilidade Física Prejudicada" em idosos: uma contribuição. Acta Paul Enferm 2002; 15(4):66-72.

(10) Araújo LAO, Santana RF, Bachion MM. Mobilidade Física Prejudicada em idosos: fatores relacionados e características definidoras. Rev Bras Enferm 2002; 55(1):19-25.
(11) Santana RF, Araújo LAO, Bachion MM. Levantamento do "Mobilidade física prejudicada" em moradores de um condomínio residencial para idosos. Livro resumo do $52^{\circ}$ Congresso Brasileiro de Enfermagem, 2000, 14-19 out. Recife-Olinda. Recife: ABEn-PE; 2000. p. 581.

(12) Ornelas DC, Pinto SM, Lappann-Boti NC. Programa saúde da família e seus desafios. In: Anais do $3^{\circ}$ Fórum Mineiro de Enfermagem; 2002, out 24-26; Uberlância. Uberlândia: Universidade Federal de Uberlândia; . 2002. p. 237.

(13) Ministério da Saúde. Conselho Nacional de Saúde. Resolução 196/96. Brasília; 1996.

(14) Cantera IR, Domingo PL. Guia prático de enfermagem: geriatria. Rio de Janeiro: McGraw Hill; 2000.

(15) Sgambatti MS. Cuidando da higiene e do vestuário do idoso no domicílio. In: Duarte YAO, Diogo MJD. Atendimento domiciliar: um enfoque gerontológico. São Paulo: Atheneu; 2000. p.249-55.

(16) Marucci MFN. Alimentação e hidratação: cuidados específicos e sua relação com o contexto familiar. In: Duarte YAO, Diogo MJD. Atendimento domiciliar: um enfoque gerontológico. São Paulo: Atheneu; 2000. p. 223-35.

(17) Ceolin MF Sono e repouso. In: Duarte YAO, Diogo MJD. Atendimento domiciliar: um enfoque gerontológico. São Paulo: Atheneu; 2000. p.349-64.
Diagnósticos de Enfermagem do Padrão Mover em idosos de uma comunidade atendida pelo Programa Saúde da Família
Correspondência: Lorena Ap. O. Araújo Rua Dona Firmina, Qd. Área Residencial Monte Verde, acesso MV 09, casa 17, Sítio dos Ipês - Goiânia CEP - 74680-190 - GO 